\title{
CRP and SAA1 Haplotypes Are Associated with Both C-Reactive Protein and Serum Amyloid A Levels: Role of Suppression Effects
}

\author{
Yu-Lin Ko, ${ }^{1,2,3}$ Lung-An Hsu, ${ }^{4}$ Semon Wu, ${ }^{1,5}$ Ming-Sheng Teng, ${ }^{1}$ and Hsin-Hua Chou ${ }^{2}$ \\ ${ }^{1}$ Department of Research, Taipei Tzu Chi Hospital, Buddhist Tzu Chi Medical Foundation, New Taipei City 23142, Taiwan \\ ${ }^{2}$ The Division of Cardiology, Department of Internal Medicine and Cardiovascular Center, Taipei Tzu Chi Hospital, \\ Buddhist Tzu Chi Medical Foundation, New Taipei City 23142, Taiwan \\ ${ }^{3}$ School of Medicine, Tzu Chi University, Hualien 97004, Taiwan \\ ${ }^{4}$ The First Cardiovascular Division, Department of Internal Medicine, \\ Chang Gung Memorial Hospital and Chang Gung University College of Medicine, Taoyuan 33305, Taiwan \\ ${ }^{5}$ Department of Life Science, Chinese Culture University, Taipei 11114, Taiwan \\ Correspondence should be addressed to Yu-Lin Ko; yulinkotw@yahoo.com.tw
}

Received 9 October 2015; Accepted 24 April 2016

Academic Editor: Elaine Hatanaka

Copyright (C) 2016 Yu-Lin Ko et al. This is an open access article distributed under the Creative Commons Attribution License, which permits unrestricted use, distribution, and reproduction in any medium, provided the original work is properly cited.

\begin{abstract}
To test the statistical association of the CRP and SAA1 locus variants with their corresponding circulating levels and metabolic and inflammatory biomarker levels by using mediation analysis, a sample population of 599 Taiwanese subjects was enrolled and five CRP and four SAA1 variants were genotyped. Correlation analysis revealed that C-reactive protein (CRP) and serum amyloid A (SAA) levels were significantly associated with multiple metabolic phenotypes and inflammatory marker levels. Our data further revealed a significant association of CRP and SAA1 variants with both CRP and SAA levels. Mediation analysis revealed that SAA levels suppressed the association between SAAl genotypes/haplotypes and CRP levels and that CRP levels suppressed the association between CRP haplotypes and SAA levels. In conclusion, genetic variants at the CRP and SAA1 loci independently affect both CRP and SAA levels, and their respective circulating levels act as suppressors. These results provided further evidence of the role of the suppression effect in biological science and may partially explain the missing heritability in genetic association studies.
\end{abstract}

\section{Introduction}

Acute-phase response is a set of immediate host inflammatory reactions that counteract challenges, such as tissue injury, infection, and trauma. Acute-phase proteins, including C-reactive protein (CRP) and serum amyloid A (SAA), are primarily produced by hepatocytes and chiefly induced by the proinflammatory cytokine interleukin-6 (IL6). In the setting of an acute-phase reaction, CRP and SAA levels can increase up to 100- to 1000-fold for a brief period and typically return to baseline levels within $2 \mathrm{wk}$ [1]. An elevated CRP level has been reported to predict the incidence of cardiovascular events and vascular mortality among apparently healthy individuals and among patients with established cardiovascular disease (CVD) [2-5]. The large JUPITER trial prospectively confirmed that CRP levels aid in tailoring statin treatments for primary prevention in patients with elevated CRP but normal low-density lipoprotein (LDL) cholesterol levels [6]. Family and twin studies have reported additive genetic factors accounting for $27 \%-40 \%$ of the variance in CRP levels [79]. Recent genome-wide association studies (GWAS) have identified multiple loci influencing CRP levels, including CRP $[6,10,11]$.

SAA is secreted by hepatocytes, macrophages, vascular smooth muscle cells, and endothelial cells [12]. SAA promotes the chemotaxis of monocytes and neutrophils and plays critical roles in a wide range of functions including cholesterol transport, high-density lipoprotein (HDL) metabolism, and 
host defense $[13,14]$. Furthermore, SAA induces, promotes, or influences susceptibility to several chronic diseases such as atherosclerosis and its clinical complications [15-18]. Twin studies have suggested a substantial genetic contribution of baseline SAA levels, with heritability estimates of $49 \%-67 \%$ [9]. GWAS have reported that the chromosome region at 11p15.5-p13, which includes the $S A A$ family, accounts for most of the explained variance in circulating SAA levels [19]. The SAA1 single nucleotide polymorphism (SNP) rs12218 was found to be associated with multiple atherosclerotic CVDs and related risk factors [20-23]. Mediation analysis suggested a suppression effect of soluble E-selectin (sE-selectin) on the association between $A B O$ genotypes and triglyceride to HDL cholesterol ratios [24]. Gene-centric analysis identified variants associated with pathways shared by different inflammatory biomarkers [25]. Thus, using pathway and mediation analysis is crucial to further elucidating the genetic determinants of inflammatory marker levels, which may be one of the reasons for the missing heritability in GWAS. Therefore, we tested the clinical and biomarker correlates of CRP and SAA levels and the statistical association of CRP and $S A A$ locus variants with the circulating levels of metabolic and inflammatory biomarkers by using mediation analysis in a Taiwanese sample population.

\section{Participants and Methods}

2.1. Study Population. This study was approved by the institutional review board of Taipei Tzu Chi Hospital, Buddhist Tzu Chi Medical Foundation (IRB number: 02-XD38-089). A total of 599 Han Chinese subjects (315 men and 284 women with mean ages of $46.1 \pm 10.0$ and $46.8 \pm 9.9 \mathrm{y}$, resp.) were recruited during routine health examinations between October 2003 and September 2005 at Chang Gung Memorial Hospital. All of the participants provided written informed consent. The subjects responded to a questionnaire on their medical history and lifestyle characteristics and underwent a physical examination that involved measurement of height, weight, waist and hip circumference, and blood pressure (BP) in the sitting position after $15 \mathrm{~min}$ of rest. Fasting blood samples were obtained from each subject. Exclusion criteria included an age younger than $18 \mathrm{y}$, CRP levels of above $10 \mathrm{mg} / \mathrm{L}$, a history of myocardial infarction, stroke, or transient ischemic attack, cancer, and current renal or liver disease. The clinical characteristics and biometrics of the study population are summarized in Table 1. Hypertension, obesity, and the current smoking status were defined as previously reported [27].

2.2. Genomic DNA Extraction and Genotyping. Genomic DNA was extracted as reported previously [24]. Nine SNPs around the CRP and SAA1 loci were selected for this study (see Supplementary Table 1 in Supplementary Material available online at http://dx.doi.org/10.1155/2016/5830361). Genotyping was performed using PCR, followed by restriction enzyme digestion or using TaqMan SNP genotyping assays obtained from Applied Biosystems (ABI, Foster City, CA, USA).
2.3. Laboratory Examinations and Assays. The laboratory examinations and assays were performed as described previously [27, 28]. Most markers, including serum CRP, SAA, soluble intercellular adhesive molecule (sICAM1), soluble vascular cell adhesive molecule (sVCAM1), sEselectin, adiponectin, matrix metalloproteinase-9 (MMP-9), and plasma monocyte chemotactic protein-1 (MCP-1), were measured using a sandwich enzyme-linked immunosorbent assay (ELISA) developed in-house. All in-house kits showed good correlation with commercially available ELISA kits. Circulating serum resistin, lipocalin-2, MMP-2 and plasma MMP-1, soluble P-selectin (sP-selectin), soluble tumor necrosis factor receptor II (sTNFRII), and interleukin-6 (IL6) were measured using commercially available ELISA kits from $\mathrm{R} \& \mathrm{D}$ (Minneapolis, MN, USA).

2.4. Statistical Analysis. The chi-square test was used for testing to compare categorical variables of diabetes mellitus and smoking. The clinical characteristics that were continuous variables are expressed as means \pm SDs and were tested using a two-sided $t$-test or analysis of variance (ANOVA). Pearson correlation coefficients $(r)$ were calculated to determine the association between CRP or SAA levels and clinical and biochemical factors with the adjustment of age and sex. Furthermore, a general linear model was applied to capture the major effect of each polymorphism on clinical and biochemical variables with BMI, age, gender, and smoking status as confounding covariates. We also used dominant models for numeric association test after recoding our SNPs from categorical variables to continuous variables, such as 0,1 of a particular allele. A value of $P<0.05$ using two-sided tests was considered statistically significant. All the above calculations were performed with standard statistical SPSS 12 software (SPSS, Chicago, IL, USA). Golden Helix SVS Win32 7.3.1 software was used to analyze the deviation from the HardyWeinberg equilibrium and to estimate the linkage disequilibrium between polymorphisms. Values of HDL cholesterol, LDL cholesterol, total cholesterol, triglyceride, CRP, SAA, sICAM1, sVCAM1, sE-selectin, sP-selectin, MMP-1, MMP2, MMP-9, MCP-1, sTNFRII, and IL6 were logarithmically transformed prior to statistical analysis to adhere to a normality assumption. The Bonferroni method was used to correct for multiple comparisons where applicable. To explore the mediating effects of SAA levels on the relationship between the SAA1 genotypes/haplotypes and CRP levels, and vice versa for the CRP genotypes/haplotypes, a conceptual model was hypothesized for the test, and four criteria were suggested for evaluating the mediating and suppression effects [24]. For example, in criterion one, independent variable (SAA1 genotypes/haplotypes) must predict the mediator (SAA levels). In criterion two, the mediator must predict the dependent variable when adjusting for independent variable (CRP level). The mediation effect was calculated as the product of the two regression coefficients from criterion one and criterion two, which reflected the intermediate pathways from independent variable to mediator and in turn to dependent variable. The regression coefficient relating independent variable to dependent variable adjusting for the mediator was expressed as direct effect. For criterion three, the total effect, expressed 
TABLE 1: Baseline characteristics of the studied subjects.

\begin{tabular}{|c|c|c|c|c|}
\hline & Total & Men & Women & $P$ value \\
\hline Number & 599 & 315 & 284 & \\
\hline Age (years) & $46.1 \pm 10.0$ & $45.5 \pm 10.0$ & $46.8 \pm 9.9$ & 0.125 \\
\hline Systolic BP (mmHg) & $113.1 \pm 16.2$ & $114.1 \pm 14.4$ & $112.0 \pm 17.9$ & 0.127 \\
\hline Diastolic BP (mmHg) & $75.0 \pm 10.0$ & $76.9 \pm 9.7$ & $73.1 \pm 9.9$ & $<0.001$ \\
\hline Total cholesterol (mg/dL) & $199.0 \pm 36.4$ & $201.3 \pm 36.9$ & $196.5 \pm 35.8$ & 0.108 \\
\hline HDL cholesterol (mg/dL) & $55.3 \pm 14.3$ & $49.8 \pm 12.0$ & $61.3 \pm 14.2$ & $<0.001$ \\
\hline LDL cholesterol (mg/dL) & $116.2 \pm 32.9$ & $118.8 \pm 33.9$ & $113.3 \pm 31.5$ & 0.041 \\
\hline Triglyceride (mg/dL) & $142.9 \pm 119.5$ & $173.4 \pm 148.6$ & $109.5 \pm 60.6$ & $<0.001$ \\
\hline Body mass index $\left(\mathrm{kg} / \mathrm{m}^{2}\right)$ & $24.3 \pm 3.4$ & $25.0 \pm 3.1$ & $23.6 \pm 3.6$ & $<0.001$ \\
\hline Diabetes mellitus (\%) & 5.0 & 5.7 & 4.2 & 0.404 \\
\hline Current smokers (\%) & 19.5 & 33.7 & 3.9 & $<0.001$ \\
\hline Fasting plasma glucose $(\mathrm{mg} / \mathrm{dL})$ & $96.4 \pm 22.6$ & $99.1 \pm 26.0$ & $93.5 \pm 17.7$ & 0.003 \\
\hline Fasting serum insulin $(\mu \mathrm{U} / \mathrm{mL})$ & $9.2 \pm 4.8$ & $9.8 \pm 5.6$ & $8.5 \pm 3.7$ & 0.001 \\
\hline HOMA-IR index & $2.2 \pm 1.4$ & $2.4 \pm 1.6$ & $2.0 \pm 1.1$ & $<0.001$ \\
\hline QUICKI & $0.35 \pm 0.02$ & $0.34 \pm 0.03$ & $0.35 \pm 0.02$ & $<0.001$ \\
\hline Adiponectin (mg/L) & $7.2 \pm 5.2$ & $5.5 \pm 4.0$ & $9.1 \pm 5.7$ & $<0.001$ \\
\hline Resistin (ng/mL) & $18.5 \pm 14.4$ & $17.7 \pm 12.0$ & $19.4 \pm 16.5$ & 0.137 \\
\hline Lipocalin-2 (ng/mL) & $79.9 \pm 52.1$ & $83.1 \pm 59.8$ & $76.5 \pm 42.0$ & 0.104 \\
\hline CRP (mg/L) & $1.1 \pm 1.4$ & $1.1 \pm 1.4$ & $1.2 \pm 1.4$ & 0.186 \\
\hline Fibrinogen $(\mathrm{mg} / \mathrm{dL})$ & $262.6 \pm 68.3$ & $260.3 \pm 70.3$ & $265.1 \pm 66.1$ & 0.389 \\
\hline sE-selectin $(\mathrm{ng} / \mathrm{mL})$ & $53.2 \pm 25.2$ & $59.8 \pm 26.0$ & $45.8 \pm 22.0$ & $<0.001$ \\
\hline sP-selectin $(\mathrm{ng} / \mathrm{mL})$ & $138.4 \pm 115.4$ & $152.9 \pm 131.1$ & $122.4 \pm 92.8$ & 0.001 \\
\hline $\mathrm{SAA}(\mathrm{mg} / \mathrm{L})$ & $5.3 \pm 11.1$ & $5.9 \pm 13.3$ & $4.7 \pm 8.0$ & 0.403 \\
\hline sICAM1 (ng/mL) & $240.0 \pm 110.9$ & $241.7 \pm 108.9$ & $238.1 \pm 113.2$ & 0.730 \\
\hline sVCAM1 (ng/mL) & $491.0 \pm 132.8$ & $493.9 \pm 150.0$ & $487.7 \pm 110.9$ & 0.706 \\
\hline MMP1 (pg/mL) & $470.1 \pm 1163.3$ & $339.5 \pm 550.5$ & $614.0 \pm 1573.7$ & 0.689 \\
\hline MMP2 (ng/mL) & $127.1 \pm 40.8$ & $124.0 \pm 41.2$ & $130.5 \pm 40.1$ & 0.053 \\
\hline MMP-9 (ng/mL) & $142.0 \pm 111.4$ & $152.9 \pm 114.9$ & $129.8 \pm 106.2$ & 0.012 \\
\hline $\mathrm{MCP} 1(\mathrm{pg} / \mathrm{mL})$ & $73.4 \pm 58.7$ & $78.7 \pm 66.7$ & $67.5 \pm 48.0$ & 0.006 \\
\hline sTNFRII $(\mathrm{pg} / \mathrm{mL})$ & $3262.4 \pm 932.4$ & $3322.7 \pm 978.8$ & $3195.9 \pm 875.3$ & 0.093 \\
\hline IL6 (pg/mL) & $3.95 \pm 7.28$ & $4.00 \pm 8.28$ & $3.90 \pm 5.98$ & 0.127 \\
\hline Creatinine $(\mathrm{mg} / \mathrm{dL})$ & $1.0 \pm 0.5$ & $1.1 \pm 0.5$ & $0.8 \pm 0.4$ & $<0.001$ \\
\hline $\mathrm{eGFR}\left(\mathrm{mL} / \mathrm{min} / 1.73 \mathrm{~m}^{2}\right)$ & $83.9 \pm 20.6$ & $86.7 \pm 20.8$ & $80.63 \pm 19.8$ & 0.001 \\
\hline
\end{tabular}

BP, blood pressure; HDL, high-density lipoprotein; LDL, low-density lipoprotein; HOMA-IR, homeostasis model assessment of insulin resistance; QUICKI, quantitative insulin sensitivity check index; CRP, C-reactive protein; SAA, serum amyloid A; sE-selectin, soluble E-selectin; sP-selectin, soluble P-selectin; sICAM1, soluble intercellular adhesive molecule 1; sVCAM1, soluble vascular cell adhesive molecule 1; MMP-1, matrix metalloproteinase-1; MMP-2, matrix metalloproteinase-2; MMP-9, matrix metalloproteinase-9; MCP-1, monocyte chemotactic protein-1; sTNFR2, soluble tumor necrosis factor-alpha receptor 2; IL6, interleukin-6; eGFR, estimated glomerular filtration rate. Continuous variables are presented as mean \pm standard deviation. HDL-C, LDL-C, total cholesterol, triglyceride, CRP, SAA, sICAM1, sVCAM1, sE-selectin, sP-selectin, MMP-1, MMP-2, MMP-9, YKL-40, MCP-1, and sTNFRII values were logarithmically transformed before statistical testing to meet the assumption of normal distributions; however, the untransformed data are shown. BP levels and lipid variables were analyzed with the exclusion of subjects using antihypertensive drugs and/or lipid-lowering agents, respectively. Fasting plasma glucose and insulin, QUICKI, and HOMA-IR index were analyzed with the exclusion of antidiabetic medications.

as the effect of independent variable on dependent variable, can be obtained by summation of direct and mediation (indirect) effects. In criterion four, the mediation effect must be significant using the procedure outlined by Sobel [26, 29]. A suppression effect may be indicated in a situation when the direct effect is larger than the total effect [30]. In this situation, the direct and indirect effects often have fairly similar magnitudes and opposite signs, which may entirely or partially cancel each other out and result in zero or a nonzero but insignificant total effect [31]. The $\beta$ coefficients and standard errors from the model above were further used to conduct a Sobel test for mediation [32]. The Sobel test was performed using a tool for mediation tests (http://www.quantpsy.org/sobel/sobel.htm), in which the null hypothesis $H_{0}: \alpha \beta=0$ is tested. The test statistic $S$, which is approximately distributed as $Z$ (2), is obtained by dividing the estimated mediation effect $(\alpha \beta)$ by the standard error $(\delta)$ in (1). The reported $P$ values are drawn from the unit normal distribution under the assumption of a $Z$ value of the hypothesis that the mediated effect equals zero in the 
population. \pm 1.96 are the critical values of the test ratio which contain the central $95 \%$ of the unit normal distribution:

$$
\begin{aligned}
\delta_{\alpha \beta}^{2} & =\delta_{\alpha}^{2} \beta^{2}+\delta_{\beta}^{2} \alpha^{2} \\
Z & =\frac{\alpha \beta}{\operatorname{SQRT}\left(\delta_{\alpha}^{2} \beta^{2}+\delta_{\beta}^{2} \alpha^{2}\right)},
\end{aligned}
$$

where SQRT is square root.

\section{Results}

3.1. Clinical and Biochemical Characteristics. A summary of demographic features, clinical profiles, and biomarker levels for the health examination participants is provided in Table 1. No significant deviation from the Hardy-Weinberg equilibrium was detected for the studied polymorphisms. All of the studied polymorphisms in the same chromosomal region were in strong pairwise linkage disequilibrium (Supplementary Tables 2 and 3 ).

\subsection{Association of CRP and SAA Levels with Clinical Parame-} ters and Other Biomarker Levels. Circulating CRP levels were positively associated with approximately all anthropometric and metabolic traits, except for the QUICKI index and HDL cholesterol level, in which a negative association was observed, and the LDL cholesterol and urinary ACR levels, in which no significant association was observed (Table 2). By contrast, SAA levels had a similar but less significant trend of the associations, having no significant association with waisthip ratios, eGFR, fasting plasma glucose, HDL cholesterol levels, and urinary ACR. Regarding inflammatory markers and adipokine levels, CRP levels were positively associated with circulating SAA, fibrinogen, sE-selectin, sTNFRII, IL6, leptin, and lipocalin-2 levels and negatively associated with adiponectin levels, whereas SAA levels were positively associated with circulating CRP, fibrinogen, IL6, and leptin levels.

3.3. Association of CRP and SAA1 Genotypes/Haplotypes with $C R P$ and $S A A$ Levels. After adjusting for clinical covariates, significant association of three $S A A 1$ polymorphisms with SAA levels was observed using an additive inheritance model (Table 3). Using a dominant inheritance model, a minor allele of rs4638289 was found to be associated with a higher SAA level $\left(P=7.48 \times 10^{-25}\right)$, whereas minor alleles of rs11024591 and rs7131332 were found to be associated with a lower SAA level $\left(P=1.28 \times 10^{-16}\right.$ and $P=$ $1.93 \times 10^{-16}$, resp.). Using haplotype analysis, two haplotypes (GATT and AAAC) inferred from four SNPs were found to be associated with SAA levels $\left(P=4.15 \times 10^{-28}\right.$ and $P=1.20 \times 10^{-27}$, resp.) (Table 4 ). The association between CRP genotypes/haplotypes and CRP levels has been reported previously [33]. In this study, we excluded subjects aged younger than $18 \mathrm{y}$ or had CRP levels above $10 \mathrm{mg} / \mathrm{L}$, and the results were similar to those of previous reports, with the exception of no significant association of the rs1800947 genotypes with CRP levels (Table 3).
3.4. Association of CRP and SAA1 Genotypes/Haplotypes with Clinical Parameters and Other Biomarker Levels. Association of CRP and SAA1 genotypes/haplotypes with various clinical parameters and biomarker levels is shown in Supplementary Tables 4-7. After further adjusting for SAA levels, a significant association of CRP levels with rs4638289, rs7131332, and rs11024591 was observed in the additive inheritance model $\left(P=2.48 \times 10^{-5}, P=0.040\right.$, and $P=0.016$, resp. $)$ and with rs4638289 in dominant inheritance model $\left(P=2.41 \times 10^{-5}\right)$. Adjustment of circulating SAA and CRP levels revealed that the $S A A 1$ haplotype $A A A C$ was associated with lower CRP levels $\left(P=1.91 \times 10^{-4}\right)$ and that the $C R P$ haplotype $A A G C G$ was associated with lower SAA levels $(P=0.046)$. Subgroup analysis of SAA quartiles revealed at least a trend of lower CRP levels with the minor allele of the rs4638289 genotypes in each SAA quartile $(P=0.008, P=0.002, P=0.023$, and $P=0.275$, separately). By contrast, a significant increase in the minor allele frequencies of the rs4638289 genotypes was observed in the higher SAA quartiles $\left(P=9.39 \times 10^{-25}\right)$, which illustrated no significant association between CRP levels and rs4638289 genotypes when all of the subjects were pooled $(P=0.733)$ (Figure 1$)$.

3.5. Mediation Analysis of Suppression Effects. Four criteria were applied to establish mediation and suppression effects. $S A A 1$ and $C R P$ genotypes/haplotypes were analyzed (Table 5). In brief, the $S A A 1$ genotypes/haplotypes were significantly associated with SAA levels (criterion 1), which in turn had significant positive effects on CRP levels (criterion 2). The total effect of SAA1 genotypes/haplotypes on CRP levels was $-0.002,-0.006,-0.006$, and 0.054 with all $P$ values nonsignificant (criterion 3). Sobel tests for mediation of the results of the corresponding CRP levels showed $z=9,6,6.67$, and 8.02 (all $P<10^{-8}$ ) (criterion 4). Moreover, the direct effects $\left(\gamma^{\prime}\right)$ of the SAA1 genotypes/haplotypes on CRP levels were greater than their total effects $\left(\alpha \beta+\gamma^{\prime}\right)$ and had similar magnitudes as mediation effects but opposite signs $(\alpha \beta)$, demonstrating a suppression effect in this model (Figure 2). The suppression effects of CRP levels on the association between the CRP haplotype AAGCG and SAA levels, of the criteria, were also observed.

\section{Discussion}

This investigation involved analyzing the association of CRP/SAA1 SNPs and CRP/SAA levels with various clinical parameters and biomarker levels. As predicted, both CRP and SAA levels correlated with multiple metabolic phenotypes and inflammatory marker levels, suggesting their crucial roles in atherosclerotic processes. Similar to the results of previous studies, our data showed a strongly significant association between $C R P / S A A 1$ SNPs and their respective circulating levels. By adjusting their individual circulating levels further, we also found $S A A$ and $C R P$ variants to be significantly associated with circulating CRP and SAA levels, respectively. Mediation analysis revealed that SAA levels have a suppression effect on the association between SAA1 genotypes/haplotypes and CRP levels, and CRP levels have 


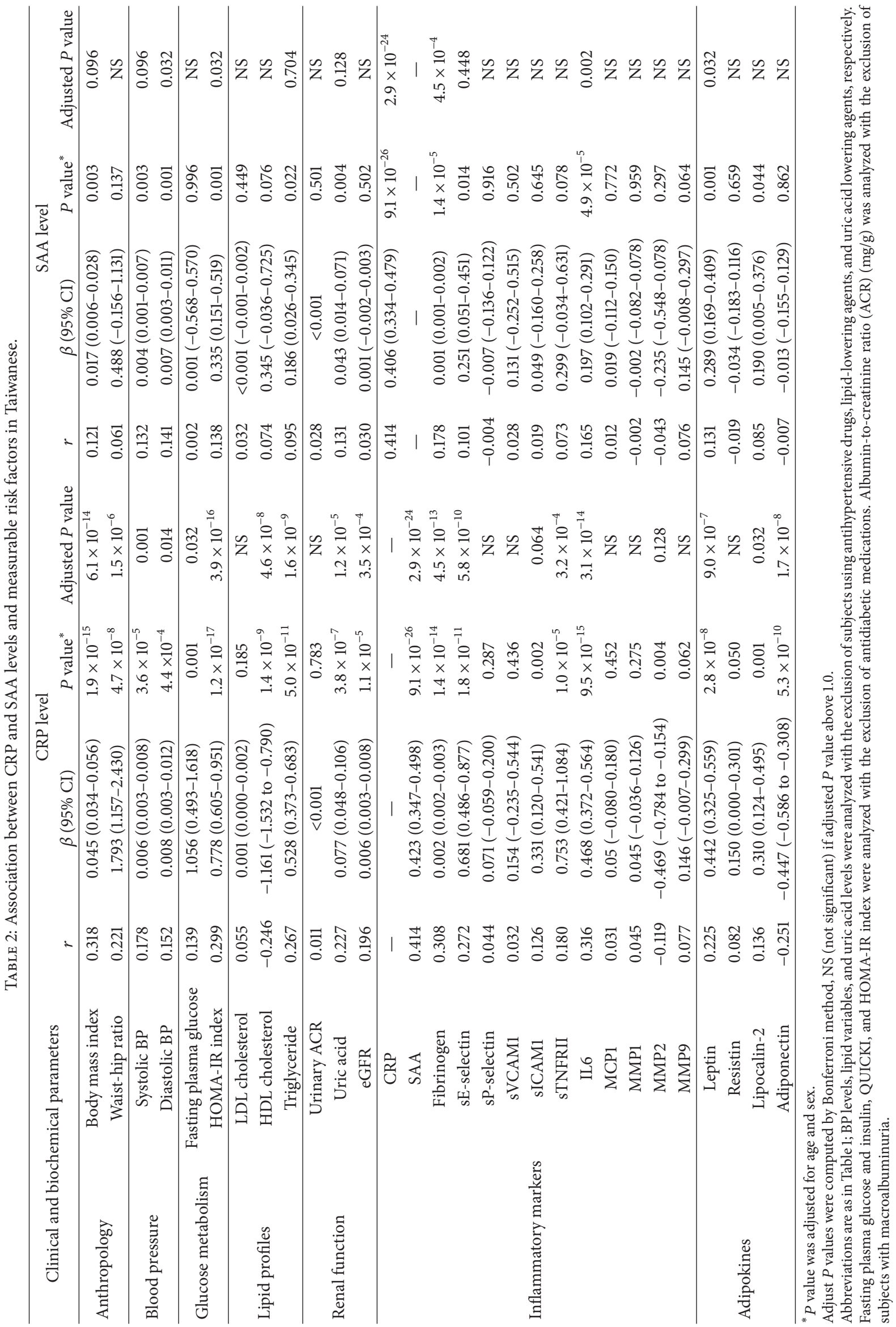




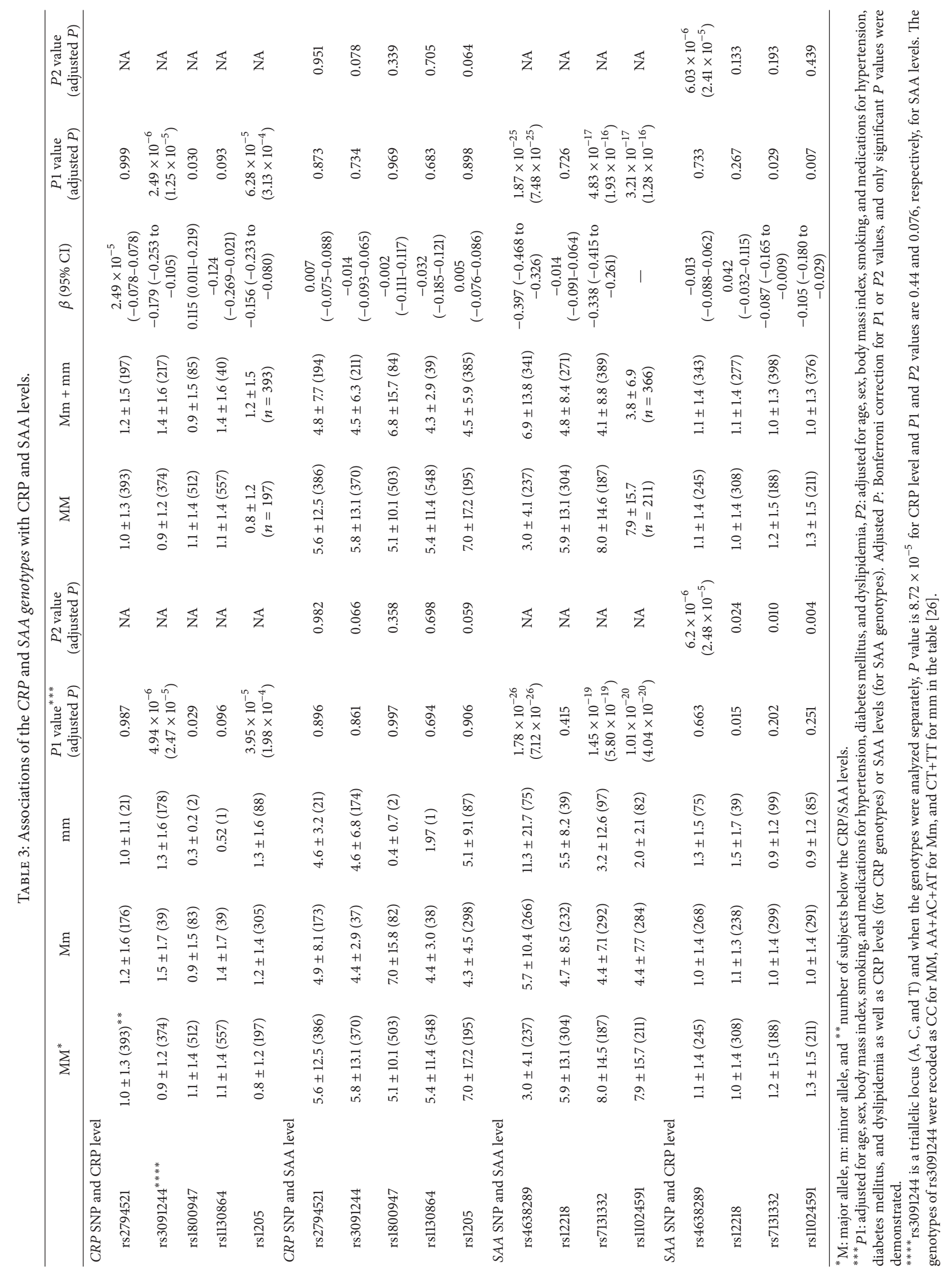




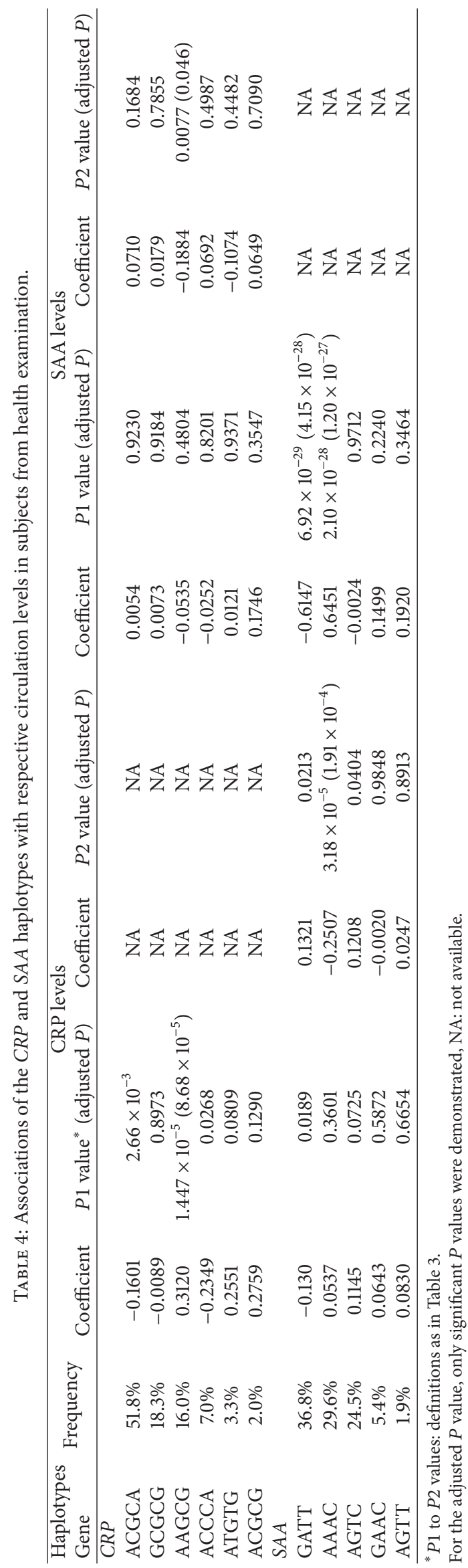


TABLE 5: Mediation test of CRP and SAA levels in the association of CRP and SAA1 genotypes/haplotypes with SAA and CRP levels.

\begin{tabular}{|c|c|c|c|c|c|}
\hline & \multicolumn{4}{|c|}{$S A A$ variants for CRP level } & \multirow{2}{*}{$\begin{array}{c}\text { CRP haplotype for SAA level } \\
\text { AAGCG }\end{array}$} \\
\hline & rs4638289" & rs7131332 $2^{\#}$ & rs11024591 ${ }^{\#}$ & AAAC & \\
\hline \multicolumn{6}{|l|}{ Criterion 1} \\
\hline \multicolumn{6}{|l|}{$\alpha$} \\
\hline Regression coefficient & -0.040 & -0.044 & -0.047 & 0.645 & 0.312 \\
\hline Standard error & 0.004 & 0.005 & 0.005 & 0.055 & 0.071 \\
\hline$P$ value & $3.87 \times 10^{-26}$ & $1.79 \times 10^{-19}$ & $7.37 \times 10^{-20}$ & $2.10 \times 10^{-28}$ & $1.45 \times 10^{-5}$ \\
\hline \multicolumn{6}{|l|}{ Criterion 2} \\
\hline \multicolumn{6}{|l|}{$\beta$} \\
\hline Regression coefficient & 0.460 & 0.420 & 0.424 & 0.460 & 0.429 \\
\hline Standard error & 0.041 & 0.040 & 0.040 & 0.041 & 0.040 \\
\hline$P$ value & $7.43 \times 10^{-27}$ & $1.23 \times 10^{-23}$ & $5.24 \times 10^{-24}$ & $2.28 \times 10^{-26}$ & $2.73 \times 10^{-24}$ \\
\hline \multicolumn{6}{|l|}{$\gamma^{\prime}$} \\
\hline Regression coefficient & 0.017 & 0.012 & 0.014 & -0.251 & -0.188 \\
\hline Standard error & 0.004 & 0.005 & 0.005 & 0.060 & 0.070 \\
\hline$P$ value & $1.24 \times 10^{-5}$ & 0.014 & 0.007 & $3.18 \times 10^{-5}$ & 0.008 \\
\hline \multicolumn{6}{|l|}{ Criterion 3} \\
\hline \multicolumn{6}{|l|}{$\alpha \beta+\gamma^{\prime}$} \\
\hline Regression coefficient & -0.002 & -0.006 & -0.006 & 0.054 & -0.054 \\
\hline Standard error & 0.004 & 0.005 & 0.005 & 0.059 & 0.076 \\
\hline$P$ value & 0.555 & 0.187 & 0.220 & 0.360 & 0.480 \\
\hline \multirow{2}{*}{\multicolumn{6}{|c|}{$\begin{array}{c}\text { Criterion } 4 \\
\alpha \beta\end{array}$}} \\
\hline & & & & & \\
\hline Regression coefficient & -0.018 & -0.018 & -0.020 & 0.297 & 0.134 \\
\hline Standard error & 0.002 & 0.003 & 0.003 & 0.037 & 0.033 \\
\hline$P$ value (Sobel test) & $<10^{-8}$ & $<10^{-8}$ & $<10^{-8}$ & $<10^{-8}$ & $4.78 \times 10^{-5}$ \\
\hline
\end{tabular}

\# Represent $S A A$ genotypes were analyzed in additive models.

$\alpha$ : unstandardized coefficient for the association between SAA1 variants and log adiponectin levels or between CRP variants and log CRP levels. $\beta$ : unstandardized coefficient for the association between SAA and CRP levels.

Direct effect $=\gamma^{\prime}$.

Total effect $=\alpha \beta+\gamma^{\prime}$.

Mediation (indirect) effect $=\alpha \beta$.

a suppression effect on the association between CRP haplotypes and SAA levels. These results suggested the relevance of $S A A 1 / C R P$ variants as the genetic determinants of both CRP and SAA levels.

SAA1 SNP and SAA Levels. Marzi et al. reported two gene loci, 11p15.5 and 1p31, to have a considerable impact on SAA levels, which compose approximately $20 \%$ of the total estimated heritability [19]. In this study, we confirmed a highly significant association of rs4638289 with SAA levels in a Taiwanese sample population. Previous studies have shown that the SAA1 SNP rs12218 was associated with multiple atherosclerotic CVDs and related risk factors [2023]. However, the association of rs12218 with SAA levels has not been elucidated previously. Our data showed no evidence of the association of the rs12218 genotypes with SAA levels and various other metabolic or inflammatory phenotypes (Table 3 and Supplementary Table 5). Additional studies may be necessary to elucidate further the role of this SNP in different ethnic populations.
Association of CRP Variants and CRP Levels with Atherogenesis: Importance of Pathway Analysis. Compelling experimental and clinical evidence suggests a crucial role of inflammation in the initiation and progression of atherosclerosis [34]. An abundance of epidemiological data has linked circulating inflammatory biomarkers with the risk of atherosclerotic CVDs and their adverse outcomes [35]. Among the wide array of inflammatory biomarkers that have been studied, high-sensitivity CRP has received the most attention for its use in screening and risk reclassification and as a predictor of clinical response to satin therapy [36]. Our study revealed a significant association of CRP and SAA levels with approximately all of the studied atherosclerosisrelated traits, including glucose metabolism, renal function, levels of various adipokines, and inflammatory markers, as well as adiposity and BP status. Although most of the correlations were not strong, one possibility is that the study participants are relatively healthy without manifested systemic diseases. Further study in patient populations, such as those with atherosclerotic cardiovascular diseases, will 


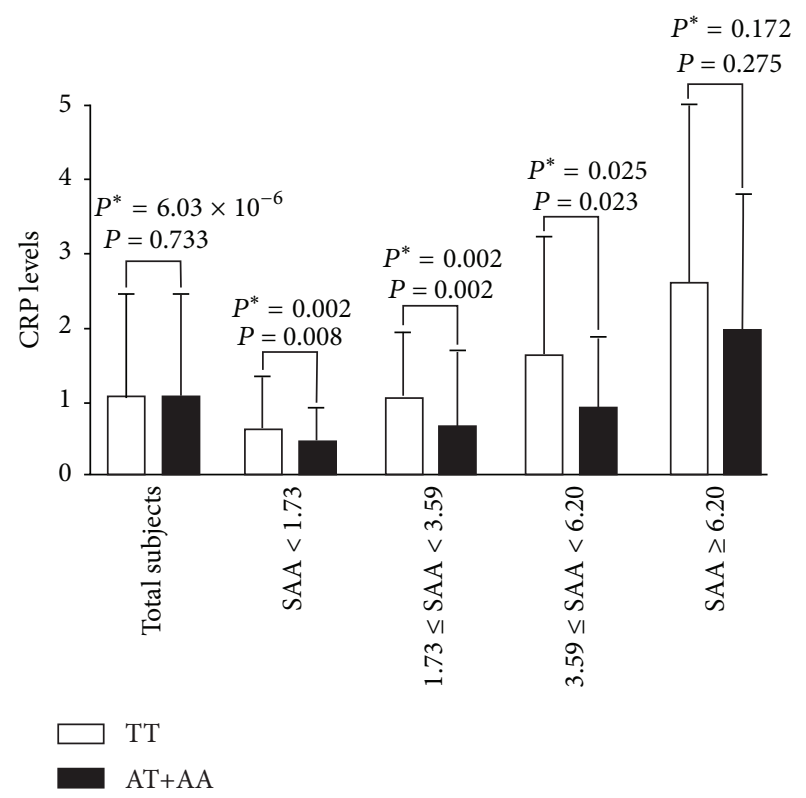

(a) Association between rs4638289 and CRP level, subgroup analysis

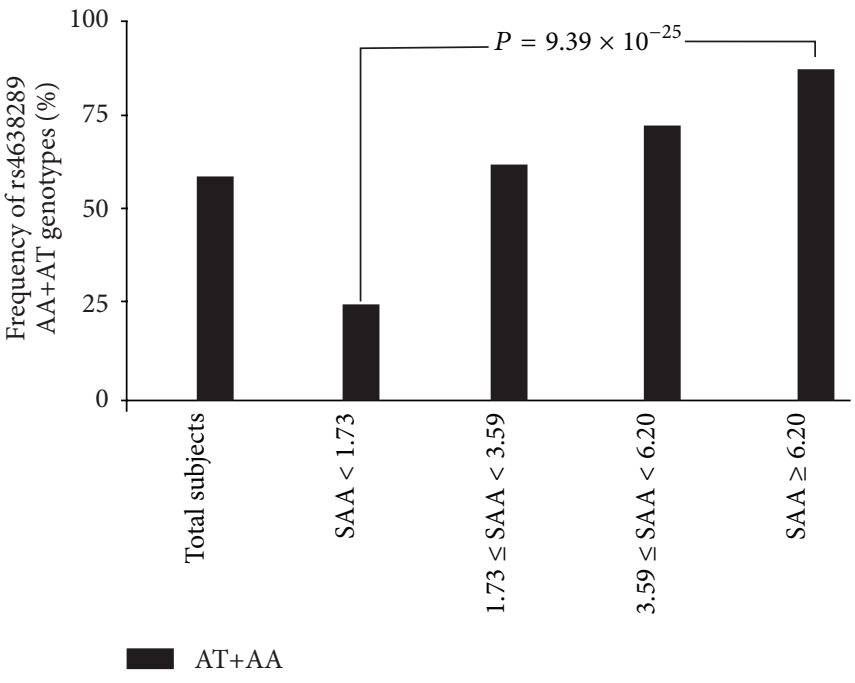

(b) Percentage of subjects in rs4638289 AA+AT

Figure 1: (a) Subgroup analysis with SAA quartiles on the association between rs4638289 genotypes and CRP levels. (b) Frequencies of subjects with $A A+A T$ genotypes of rs4638289 in each SAA quartile. $P$ value: adjusted for age, sex, body mass index, smoking, and medications for hypertension, diabetes mellitus, and dyslipidemia. $P^{*}$ value: further adjusted for SAA levels.

help us to understand whether CRP/SAA levels have a stronger association with various atherosclerotic risk factors in disease populations. These results further support the multifaceted perspective of the CRP and SAA levels affecting the pathogenesis of atherosclerosis. Recent GWAS studies have shown multiple genetic determinants of CRP and SAA levels; however, the probability of SNPs contributing much to the current approach of risk assessment, based on conventional risk factors, is low. Our data provide the first evidence suggesting the importance of CRP and SAA1 variants as genetic determinants of both CRP and SAA levels. Carty et al. showed that gene-gene interaction between rs 4638289 and genetic variants of $S A A$ regulators influences CVD [37]. Shah et al. revealed the association of interleukin6 receptor (IL6R) genotypes with both IL6 and CRP levels [25]. Thus, a paradigm shift would involve using a genecentric approach to investigating an entire pathway rather than assessing isolated mutations for providing more useful information on complex traits that involve a high number of genes and are subject to environmental regulation of gene expression and cellular phenotypes [25].

Suppression Effect and Possible Mechanisms. Suppression effects have been rarely reported in biological science. Mediation hypotheses suggest a suppression effect if the statistical removal of a mediating effect enhances the relationship between the independent and dependent variables. We previously reported that $\mathrm{sE}$-selectin levels have a suppression effect on the association between $A B O$ blood group genotypes and triglyceride to HDL cholesterol ratios [24]; this emphasizes the importance of the relationship between $\mathrm{ABO}$ blood groups and atherogenesis. In our recent study, adiponectin levels also acted as suppressors for the association between CDH13 SNPs and metabolic syndrome and metabolic phenotypes [38]. The data in our current study suggested that SAA and CRP levels act as suppressors of the association between their respective gene variants and circulating levels of other inflammatory biomarkers. The association between $S A A$ rs4638289 genotypes and CRP levels became more significant in subgroup analysis with different quartiles of SAA levels. Lower CRP levels were observed in each SAA quartile in subjects with the minor allele A of the rs4638289 genotypes, whereas higher $A A+A T$ genotype frequencies were observed with increased SAA quartiles (which are associated with higher CRP levels); this may partially explain why no significant difference was observed between rs4638289 genotypes and CRP levels when all of the studied subjects were pooled for analysis without adjusting the SAA levels. Altogether, these findings indicate the crucial role of suppression effects in biological science. Mediation analysis would elucidate the additional genetic determinants of biomarker levels and disease status.

Several possible biological mechanisms of the suppression effect may be considered. MicroRNAs have emerged as key gene regulators, including trans- or cisregulation, for diverse biological pathways in various vascular and metabolic diseases [39]. Online resources for microRNA target prediction may be helpful in searching for possible candidates linked between CRP and $S A A$ loci. It is also possible that the association of the $S A A$ polymorphism with CRP levels may be due to linkage disequilibrium with another polymorphism that is linked to CRP regulation. More importantly, the SAA 


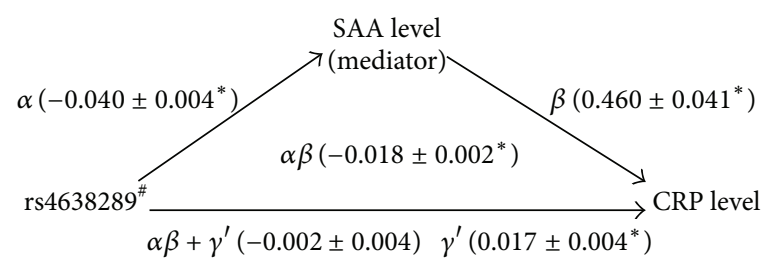

(a)

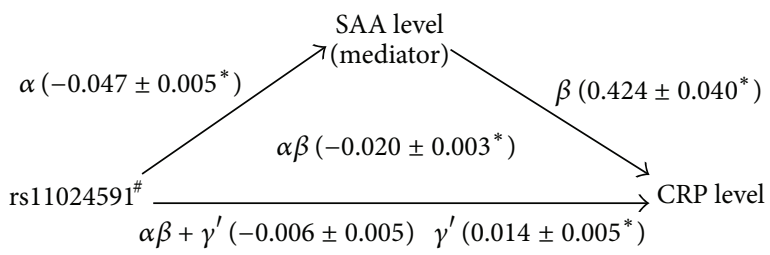

(c)

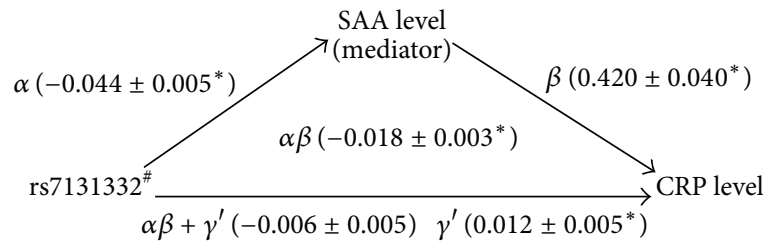

(b)

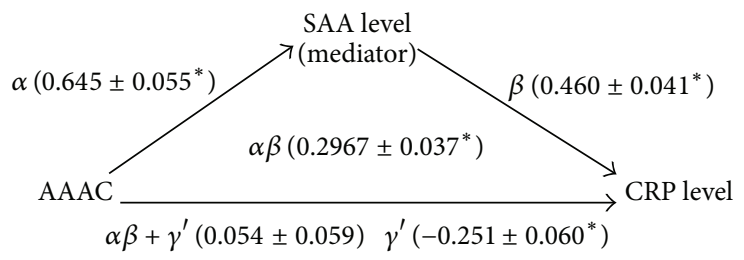

(d)

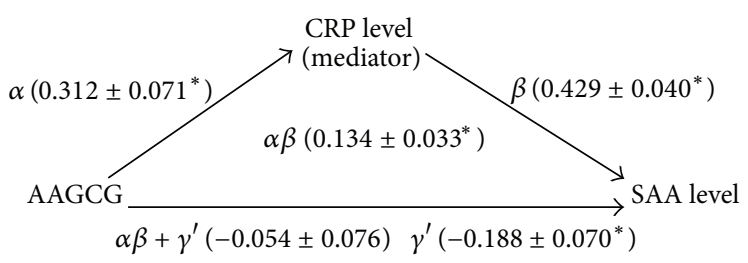

(e)

FIGURE 2: A three-variable mediation model: SAA and CRP levels as a mediator of the association between the $S A A$ and $C R P$ genotypes/haplotypes and CRP and SAA levels. Linear regression models were used to assess the following path associations: in Figure 1(a): $(\alpha)$ relationship between the SAA genotype and SAA level, $(\beta)$ relationship between SAA level and CRP level, $\left(\alpha \beta+\gamma^{\prime}\right)$ relationship between the SAA genotype and CRP level, and $\left(\gamma^{\prime}\right)$ relationship between the SAA genotype and CRP level after adjustment for SAA levels. Each estimate along the path represents the unstandardized $\beta$ coefficient from the regression model. The results indicate that, after adjustment for SAA levels, the SAA genotypes exhibited a stronger association with CRP level. The direct effects $\left(\gamma^{\prime}\right)$ of the SAA genotypes on the CRP level $(0.017)$ were greater than the total effects $\left(\alpha \beta+\gamma^{\prime}\right)(-0.002)$ and revealed similar magnitudes and opposite signs than those of the mediation effects $(\alpha \beta)(-0.018)$, suggesting significant mediation (suppression) by the SAA levels. All of the models were adjusted for age, sex, BMI, current smoking status, and medications for hypertension, diabetes mellitus, and dyslipidemia. ${ }^{*} P<0.05$. In addition, the other analysis exhibited similar suppression effects ((b), (c), (d), and (e)). "SAA genotypes.

and CRP levels are affected by multiple proinflammatory cytokines [40]. The proinflammatory cytokine IL6 is a critical mediator that induced both CRP and SAA expression in hepatocytes. IL6R gene variants have been associated with CRP levels [25]. Significant associations of circulating IL6 levels with CRP and SAA levels were noted in this investigation; however, our preliminary data revealed that the association of CRP/SAA genotypes/haplotypes with CRP and SAA levels was not affected with adjustment of IL6 levels (data not shown). Thus, further investigation involving multiple candidate targets will be necessary in the future to establish the molecular basis of the suppression effect.

Suppression Effects May Partially Explain the Missing Heritability. Recent GWAS have identified hundreds of genetic variants associated with complex human diseases and traits. However, complex inheritance can assume numerous forms and GWAS have only partially explained heritability. The missing heritability may be due to multiple factors, including a high number of variants with smaller effects yet to be found, rare variants with larger effects, which are poorly detected by available genotyping arrays, structural variants poorly captured by existing arrays, low power for detecting gene-gene and gene-environment interactions, and sequence-independent epigenetic effects including noncoding microRNAs [41, 42]. Our recent investigations revealed that mediation analysis of suppression effects may elucidate several previously unidentified associations between genetic variants in one gene and other closely associated metabolic or inflammatory phenotypes, which may in turn partially explain the missing heritability in complex diseases.

Limitation. Our study has several limitations, one of which is the relatively modest number of studied subjects. Furthermore, only 9 of the CRP and SAA1 SNPs were analyzed, and this incomplete genotyping may not represent all of the haplotypes in CRP and $S A A 1$. Another limitation of this study is its cross-sectional design, which could draw only limited inference regarding the relationships between exposure and outcome. Finally, the examined subjects were ethnically Chinese; hence, caution should be exercised when extrapolating our results to other ethnic groups.

\section{Conclusion}

Our data revealed a significant association of CRP and SAA1 variants with both CRP and SAA levels, which are highly 
correlated with multiple atherosclerosis-related traits. These results provide further evidence of the role of suppression effects in biological science and may partially explain the missing heritability in genetic association studies. Further analysis of the interrelationship between entire pathways of inflammatory gene variants and circulating levels may further elucidate the pathogenesis of atherosclerotic CVDs.

\section{Competing Interests}

The authors declare that they have no competing interests.

\section{Acknowledgments}

This study was supported by a grant from the National Science Council, Taiwan (no. NSC101-2314-B-303-023-MY3), grants from the Taipei Tzu Chi Hospital, Buddhist Tzu Chi Medical Foundation (nos. TCRD-TPE-99-07, TCRDTPE-NSC-102-01, TCRD-TPE-MOST-103-01, and TCRDTPE-103-R-2), and grants from Tzu Chi University (nos. TCIRP102001-02Y1 and TCIRP99001-04Y1) to Y.-L. Ko. The authors greatly appreciate the technical support from the Taipei Tzu Chi Hospital, Buddhist Tzu Chi Medical Foundation Core Laboratory.

\section{References}

[1] G. Marhaug, L. Hårklau, B. Olsen, G. Husby, A. Husebekk, and H. Wang, "Serum amyloid A protein in acute myocardial infarction," Acta Medica Scandinavica, vol. 220, no. 4, pp. 303306, 1986.

[2] P. M. Ridker, M. Cushman, M. J. Stampfer, R. P. Tracy, and C. H. Hennekens, "Inflammation, aspirin, and the risk of cardiovascular disease in apparently healthy men," The New England Journal of Medicine, vol. 336, no. 14, pp. 973-979, 1997.

[3] P. M. Ridker, N. Rifai, L. Rose, J. E. Buring, and N. R. Cook, "Comparison of C-reactive protein and low-density lipoprotein cholesterol levels in the prediction of first cardiovascular events," The New England Journal of Medicine, vol. 347, no. 20, pp. 1557-1565, 2002.

[4] J. Danesh, J. G. Wheeler, G. M. Hirschfield et al., "C-reactive protein and other circulating markers of inflammation in the prediction of coronary heart disease," The New England Journal of Medicine, vol. 350, no. 14, pp. 1387-1397, 2004.

[5] J. K. Pai, T. Pischon, J. Ma et al., "Inflammatory markers and the risk of coronary heart disease in men and women," The New England Journal of Medicine, vol. 351, no. 25, pp. 2599-2610, 2004.

[6] P. M. Ridker, G. Pare, A. Parker et al., "Loci related to metabolicsyndrome pathways including LEPR, HNF1A, IL6R, and GCKR associate with plasma C-reactive protein: the Women's Genome Health study," The American Journal of Human Genetics, vol. 82, no. 5, pp. 1185-1192, 2008.

[7] J. S. Pankow, A. R. Folsom, M. Cushman et al., "Familial and genetic determinants of systemic markers of inflammation: the NHLBI family heart study," Atherosclerosis, vol. 154, no. 3, pp. 681-689, 2001.

[8] L. Retterstol, L. Eikvar, and K. Berg, "A twin study of Creactive protein compared to other risk factors for coronary heart disease," Atherosclerosis, vol. 169, no. 2, pp. 279-282, 2003.
[9] A. J. MacGregor, J. R. Gallimore, T. D. Spector, and M. B. Pepys, "Genetic effects on baseline values of C-reactive protein and serum amyloid a protein: a comparison of monozygotic and dizygotic twins," Clinical Chemistry, vol. 50, no. 1, pp. 130-134, 2004.

[10] P. Elliott, J. C. Chambers, W. Zhang et al., "Genetic Loci associated with C-reactive protein levels and risk of coronary heart disease," The Journal of the American Medical Association, vol. 302, no. 1, pp. 37-48, 2009.

[11] A. Dehghan, J. Dupuis, M. Barbalic et al., "Meta-analysis of genome-wide association studies in $>80000$ subjects identifies multiple loci for C-reactive protein levels," Circulation, vol. 123, no. 7, pp. 731-738, 2011.

[12] R. L. Meek, S. Urieli-Shoval, and E. P. Benditt, "Expression of apolipoprotein serum amyloid A mRNA in human atherosclerotic lesions and cultured vascular cells: implications for serum amyloid A function," Proceedings of the National Academy of Sciences of the United States of America, vol. 91, no. 8, pp. 31863190, 1994.

[13] R. Kisilevsky and S.-P. Tam, "Acute phase serum amyloid A, cholesterol metabolism, and cardiovascular disease," Pediatric Pathology and Molecular Medicine, vol. 21, no. 3, pp. 291-305, 2002.

[14] V. L. King, J. Thompson, and L. R. Tannock, "Serum amyloid A in atherosclerosis," Current Opinion in Lipidology, vol. 22, no. 4, pp. 302-307, 2011.

[15] A. I. Fyfe, L. S. Rothenberg, F. C. DeBeer, R. M. Cantor, J. I. Rotter, and A. J. Lusis, "Association between serum amyloid A proteins and coronary artery disease: Evidence from two distinct arteriosclerotic processes," Circulation, vol. 96, no. 9, pp. 2914-2919, 1997.

[16] B. D. Johnson, K. E. Kip, O. C. Marroquin et al., "Serum amyloid A as a predictor of coronary artery disease and cardiovascular outcome in women: the National Heart, Lung, and Blood Institute-Sponsored Women's Ischemia Syndrome Evaluation (WISE)," Circulation, vol. 109, no. 6, pp. 726-732, 2004.

[17] W. Maier, L. A. Altwegg, R. Corti et al., "Inflammatory markers at the site of ruptured plaque in acute myocardial infarction: locally increased interleukin- 6 and serum amyloid a but decreased C-reactive protein," Circulation, vol. 111, no. 11, pp. 1355-1361, 2005.

[18] M. Schillinger, M. Exner, W. Mlekusch et al., "Inflammation and carotid artery-risk for atherosclerosis study (ICARAS)," Circulation, vol. 111, no. 17, pp. 2203-2209, 2005.

[19] C. Marzi, E. Albrecht, P. G. Hysi et al., "Genome-wide association study identifies two novel regions at 11p15.5-p13 and 1p31 with major impact on acute-phase serum amyloid a," PLoS Genetics, vol. 6, no. 11, Article ID e1001213, 2010.

[20] X. Xie, Y.-T. Ma, Y.-N. Yang et al., "Polymorphisms in the SAA1/2 gene are associated with carotid intima media thickness in healthy Han Chinese subjects: the Cardiovascular Risk Survey," PLoS ONE, vol. 5, no. 11, Article ID e13997, 2010.

[21] X. Xie, Y.-T. Ma, Y.-N. Yang et al., "Serum uric acid levels are associated with polymorphism in the SAA1 gene in Chinese subjects," PLoS ONE, vol. 7, no. 6, Article ID e40263, 2012.

[22] X.-L. Xu, X.-T. Sun, L. Pang et al., "Rs12218 in SAA1 gene was associated with serum lipid levels," Lipids in Health and Disease, vol. 12, no. 1, article 116, 2013.

[23] B. Y. Wang, J. Y. Hang, Y. Zhong, and S. J. Tan, "Association of genetic polymorphisms of SAA1 (rs12218) with myocardial infarction in a Chinese population," Genetics and Molecular Research, vol. 13, no. 2, pp. 3693-3696, 2014. 
[24] M.-S. Teng, L.-A. Hsu, S. Wu et al., "Mediation analysis reveals a sex-dependent association between $\mathrm{ABO}$ gene variants and TG/HDL-C ratio that is suppressed by sE-selectin level," Atherosclerosis, vol. 228, no. 2, pp. 406-412, 2013.

[25] T. Shah, D. Zabaneh, T. Gaunt et al., "Gene-centric analysis identifies variants associated with interleukin-6 levels and shared pathways with other inflammation markers," Circulation: Cardiovascular Genetics, vol. 6, no. 2, pp. 163-170, 2013.

[26] D. P. MacKinnon, "Analysis of mediating variables in prevention and intervention research," in Scientific Methods for Prevention/Intervention Research, vol. 139 of NIDA Research Monograph, pp. 127-153, DHHS, 1994.

[27] L.-A. Hsu, C.-J. Chang, S. Wu et al., "Association between functional variants of the ICAM1 and CRP genes and metabolic syndrome in Taiwanese subjects," Metabolism: Clinical and Experimental, vol. 59, no. 12, pp. 1710-1716, 2010.

[28] S. Wu, L.-A. Hsu, M.-S. Teng et al., "Association of SELE genotypes/haplotypes with sE-selectin levels in Taiwanese individuals: interactive effect of MMP9 level," BMC Medical Genetics, vol. 13, article 115, 2012.

[29] R. M. Baron and D. A. Kenny, "The moderator-mediator variable distinction in social psychological research: conceptual, strategic, and statistical considerations," Journal of Personality and Social Psychology, vol. 51, no. 6, pp. 1173-1182, 1986.

[30] M. E. Sobel, "Direct and indirect effects in linear structural equation models," Sociological Methods \& Research, vol. 15, pp. 151-176, 1987.

[31] D. P. MacKinnon, J. L. Krull, and C. M. Lockwood, "Equivalence of the mediation, confounding and suppression effect," Prevention Science, vol. 1, no. 4, pp. 173-181, 2000.

[32] M. E. Sobel, "Asymptotic confidence intervals for indirect effects in structural equation models," Sociological Methodology, vol. 13, pp. 290-312, 1982.

[33] M.-S. Teng, L.-A. Hsu, S. Wu, H.-H. Chang, H.-H. Chou, and Y.L. Ko, "Association between C-reactive protein gene haplotypes and C-reactive protein levels in Taiwanese: interaction with obesity," Atherosclerosis, vol. 204, no. 2, pp. e64-e69, 2009.

[34] P. Libby, P. M. Ridker, and A. Maseri, "Inflammation and atherosclerosis," Circulation, vol. 105, no. 9, pp. 1135-1143, 2002.

[35] E. Zakynthinos and N. Pappa, "Inflammatory biomarkers in coronary artery disease," Journal of Cardiology, vol. 53, no. 3, pp. 317-333, 2009.

[36] P. M. Ridker, E. Danielson, F. A. H. Fonseca et al., "Rosuvastatin to prevent vascular events in men and women with elevated Creactive protein," The New England Journal of Medicine, vol. 359, no. 21, pp. 2195-2207, 2008.

[37] C. L. Carty, P. Heagerty, S. R. Heckbert et al., "Association of genetic variation in serum amyloid-a with cardiovascular disease and interactions with IL6, IL1RN, IL1 $\beta$ and TNF genes in the Cardiovascular Health Study," Journal of Atherosclerosis and Thrombosis, vol. 16, no. 4, pp. 419-430, 2009.

[38] M.-S. Teng, L.-A. Hsu, S. Wu, Y.-C. Sun, S.-H. Juan, and Y.-L. Ko, "Association of $\mathrm{CDH} 13$ genotypes/haplotypes with circulating adiponectin levels, metabolic syndrome, and related metabolic phenotypes: the role of the suppression effect," PLOS ONE, vol. 10, no. 4, Article ID e0122664, 2015.

[39] A. Zampetaki and M. Mayr, "MicroRNAs in vascular and metabolic disease," Circulation Research, vol. 110, no. 3, pp. 508522, 2012.

[40] K. Schroder and J. Tschopp, “The inflammasomes," Cell, vol.140, no. 6, pp. 821-832, 2010.
[41] T. A. Manolio, F. S. Collins, N. J. Cox et al., "Finding the missing heritability of complex diseases," Nature, vol. 461, no. 7265, pp. 747-753, 2009.

[42] E. E. Eichler, J. Flint, G. Gibson et al., "Missing heritability and strategies for finding the underlying causes of complex disease," Nature Reviews Genetics, vol. 11, no. 6, pp. 446-450, 2010. 


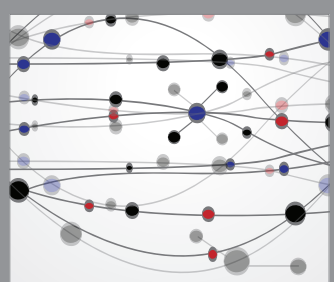

The Scientific World Journal
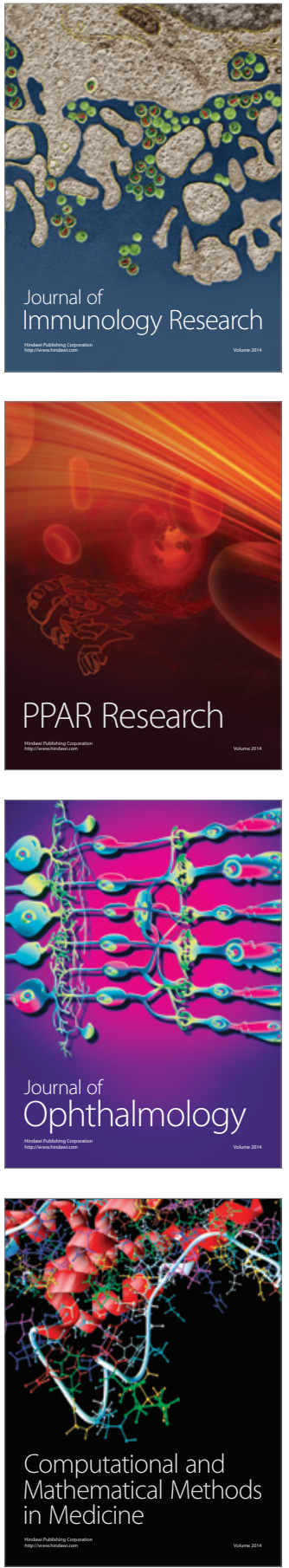

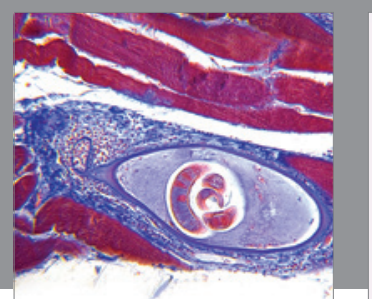

Gastroenterology Research and Practice

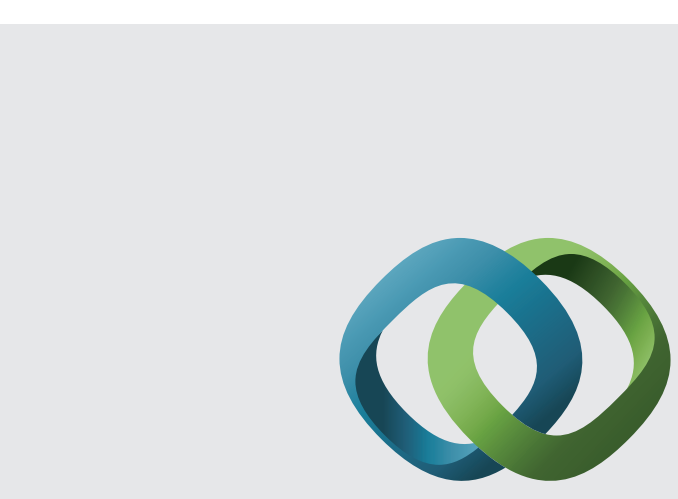

\section{Hindawi}

Submit your manuscripts at

http://www.hindawi.com
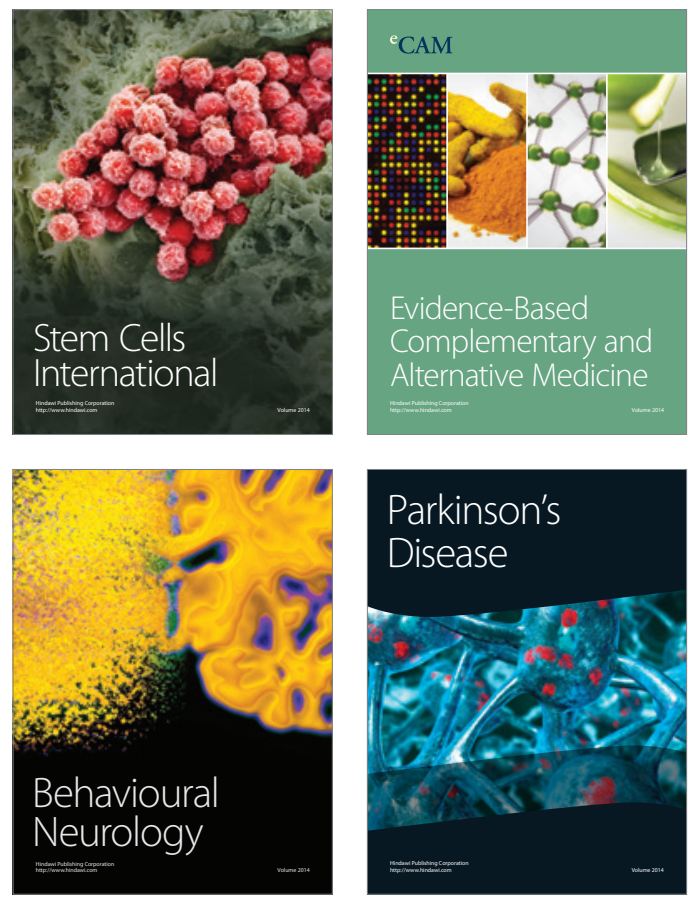
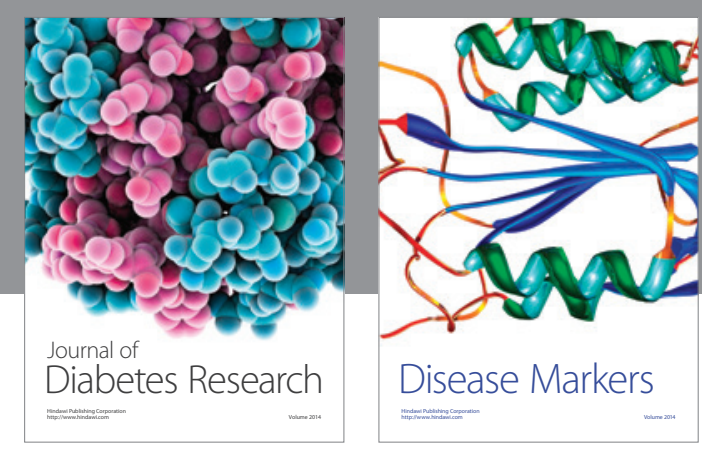

Disease Markers
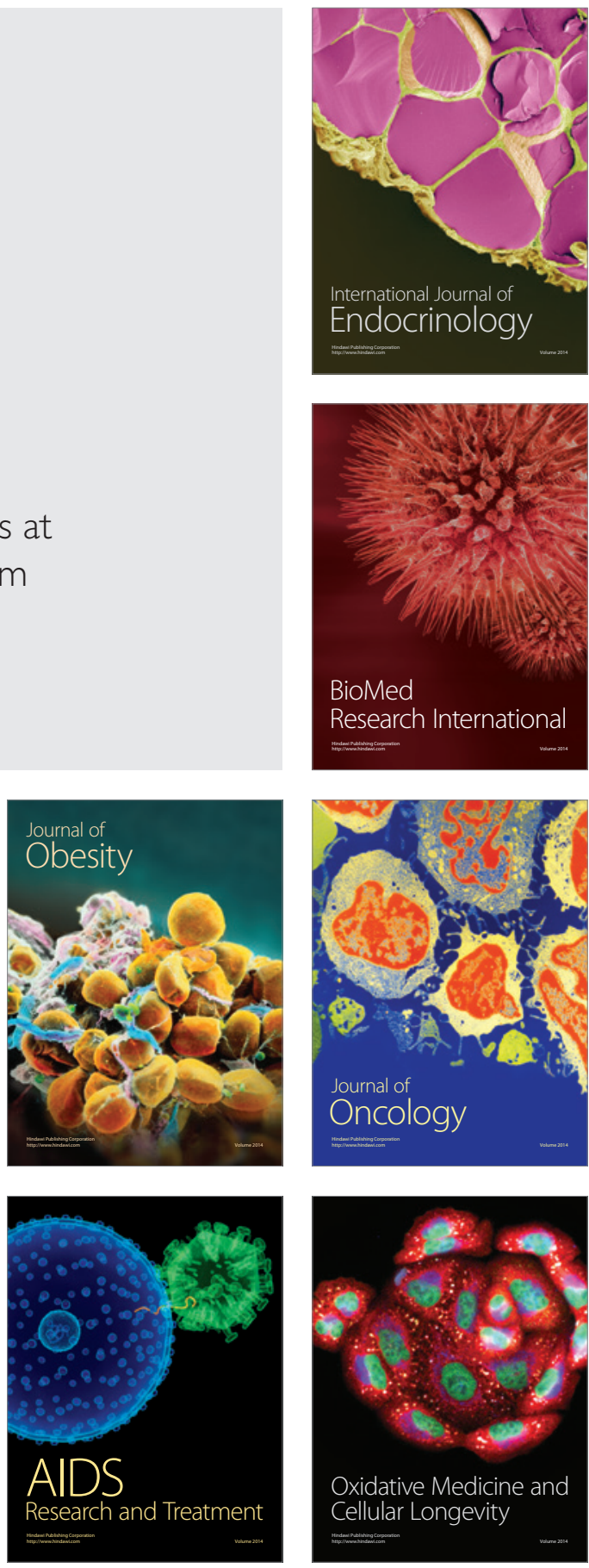